\title{
ON THE DYNAMIC ANALYSIS OF A LEONTIEF MODEL WITH FIXED CAPITAL
}

\author{
J. E. WOODS ${ }^{1}$
}

(Received 18 January 1985; revised 13 June 1985)

\begin{abstract}
In Convex Structures and Economic Theory, Nikaido analysed, inter alia, a circulatıng capital Leontief model where final demand could exhibit either proportional or non-proportional growth. This paper extends his analysis to a fixed capital model. Analogues of Nikaido's results are derived for the closed model and for the open model under balanced growth. However, the results obtained here for the open model with unbalanced growth are weaker than Nikaido's.
\end{abstract}

\section{Introduction}

In the simplest multi-sector production model, each industry has only one available process, which lasts for a common period, say a year: a typical process, the $j$ th, requires inputs of circulating capital and homogeneous labour at the beginning of a year to produce as output at the end of the year a quantity of the $j$ th commodity. The simplicity of the model is a consequence of the following: first, the question of choice of technique cannot arise, there being only one technique consisting of the process for each industry; second, there are only single-product industries, pure joint production of the wheat-and-straw or wooland-mutton variety being absent; third, neither fixed capital nor such non-produced means of production as land is required in any process, the presence of either of which would necessitate treatment in a multiple-product industries framework (as demonstrated in Chapters X and XI of Sraffa [5]); fourth, as there is a common period of production, there is no need to introduce fictitious commodities to represent work-in-progress (on this point, see Chapter 7 of Woods

\footnotetext{
${ }^{1}$ Faculty of Economics and Politics, University of Cambridge, Sidgwick Avenue, Cambridge CB3 9DD, England

(C) Copyright Australian Mathematical Society 1986, Serial-fee code 0334-2700/86
} 
[6] or Chapter 6 of Morishima [1]). In essence, we have the single-product industries, circulating capital model of Part I of Sraffa [5], the quantity side of which can be described by this equation

$$
x(t)=A x(t+1)+f(t+1),
$$

$x(t)$ being the vector of sectoral gross outputs at the end of period $t, A=\left[a_{i j}\right]$ the $n \times n$ matrix of circulating capital input coefficients, and $f(t+1)$ the vector of final demand in period $(t+1) . f(t+1)$ could represent the vector of final demand at the end of period $t$; the important point is not so much the timing of consumption-at the end of $t$, on the one hand, or during $(t+1)$, on the other-as that $f(t+1)$ is met out of $x(t)$. Production is described in (1) by a backward difference equation, the gross outputs of period $t$ being used in period $(t+1)$ to meet inter-industry and final demand requirements; indeed, the mathematical representation of production necessarily involves a backward difference equation if processes take time.

Nikaido [3] has analysed the behaviour of (1) under different specifications of the final demand vector. The purpose of this paper is to extend Nikaido's analysis by generalising the single-product industries, circulating capital model, (1), to take account of fixed capital, albeit in the simplest possible way. Recalling that a multiple-product industries framework is required for the treatment of fixed capital, we must have technology specified by an $n \times n$ input matrix $C$ and an $n \times n$ output matrix $B$ (which replace $A$ and the identity matrix respectively in (1)), on the assumption that each industry has only one process. Retaining the assumption of a common period of production, we have instead of (1)

$$
B x(t)=C x(t+1)+f(t+1) .
$$

In (1), $A$ is a commodity/commodity matrix, its typical element, $a_{i j}$, being the quanity of commodity $i$ advanced per unit of output of commodity $j$. In (2), both $C=\left[c_{i j}\right]$ and $B=\left[b_{i j}\right]$ are commodity/process matrices; $c_{i j}$ is the input and $b_{i j}$ the output of commodity $i$ per unit intensity of process $j$. Correspondingly, $x(t)$ must be interpreted as an activity vector in (2), and not as a gross output vector as in (1).

Fixed capital is treated by means of a priori specified exponential depreciation coefficients. While unsatisfactory compared with the treatment in terms of joint production $^{2}$, this approach can be justified on the grounds that the resulting model is relatively tractable. Our treatment of fixed capital permits the retention of the single-product industries assumption (i.e. that each industry produces only one final, or marketable, commodity). The elements of the matrix $B$ in (2) thus

\footnotetext{
${ }^{2}$ As in von Neumann [2] and Sraffa [5]. See also Woods [7].
} 
refer to outputs of marketable commodities and durable instruments of production. We define

$$
B=I+K,
$$

where $I=$ identity (i.e. commodity output) matrix of order $n$,

$$
K=n \times n \text { matrix of fixed capital coefficients net of depreciation. }
$$

The vector of total commodity outputs at the end of period $t$ (i.e. marketable commodities plus fixed capital goods) is given by $B x(t)=(I+K) x(t)$. The elements of $C$ refer to both circulating and fixed capital inputs. We define

$$
C=A+K
$$

where $A=n \times n$ matrix of circulating capital inputs and depreciation coefficients. If commodity $i$ is used only as a circulating capital good, it follows that $k_{\imath j}=0$ for all $j$. On the other hand, if commodity $i$ is used only as a fixed capital good, $a_{i j}$ is the depreciation coefficient of capital good $i$ when used in industry $j$; as no restriction is placed on the depreciation coefficients, it is possible that $a_{i j} \neq a_{t k}$ for $j \neq k$ (that is, capital good $i$ depreciates at different rates in industries $j$ and $k$ ) and that $a_{i j} \neq a_{h j}$ for $i \neq h$ (that is, capital goods $i$ and $h$ depreciate at different rates when employed in the same industry, $j$ ). Finally, it is possible that commodity $i$ may be used as circulating capital in one industry and fixed capital in another. This treatment of capital is the most general one available within the confines of a priori specified depreciation coefficients.

Substituting (4) and (3) into (2), we have

$$
\begin{gathered}
(I+K) x(t)=(A+K) x(t+1)+f(t+1) \text { or } \\
x(t)=A x(t+1)+K[x(t+1)-x(t)]+f(t+1) .
\end{gathered}
$$

This resembles the equation obtained in the discrete-time formulation of the Dynamic Leontief $\mathrm{Model}^{3}$, viz.

$$
x(t)=A x(t)+K[x(t+1)-x(t)]+f(t+1) .
$$

The assumption in (6), that current inputs are met from current outputs, is made for mathematical convenience, as will be seen below. If time is to be introduced into the model, it is preferable to do so in a consistent way; current inputs should be met from last period's outputs as in (5), and more generally in (2).

(5) is a non-homogeneous backward difference equation. Following the method adopted by Nikaido [3] for his analysis of the simpler model (1), we consider first of all the following homogeneous equation associated with (5)

$$
B x(t)=C x(t+1) \text { or }(I+K) x(t)=(A+K) x(t+1) .
$$

\footnotetext{
${ }^{3}$ See, for example, Chapter 5 of Woods [5].
} 
Having solved this equation in Section 2, we proceed in Section 3 to considering the solution of the complete system (5) when the final demand vector exhibits first proportional and then non-proportional growth. In general, we are following the procedure for solving forward difference equations - that is, adding a particular solution to the solution of the homogeneous equation-though it should be remembered that backward difference equations present their own particular problems.

Before proceeding with the analysis of (5), we state two fundamental assumptions

(A1) $B$ and $C$ are non-singular.

(A2) $A$ is productive and indecomposable.

(A2) is central to all production models. It means that a physical surplus can be produced so that accumulation can take place and/or a final demand vector in any proportions can be met. If (A2) did not hold, we would be dealing with either a subsistence economy or one in which larger quantities of commodities were used up than were produced (i.e. an unproductive economy). In the context of this model, productiveness is equivalent to $\lambda^{*}(A)<1, \lambda^{*}(A)$ being the Frobenius root of $A^{4}$.

\section{The homogeneous or closed system}

With $f(t+1)=\underline{0}$ in (2), we have

$$
B x(t)=C x(t+1) .
$$

Under (A1), this can be rewritten as

$$
x(t+1)=C^{-1} B x(t) \text { or } x(t)=B^{-1} C x(t+1) .
$$

However, the properties of $C^{-1} B=(A+K)^{-1}(I+K)$ or $B^{-1} C=(I+K)^{-1}(A$ $+K)$ are not evident; in particular, it is not clear that either exhibits a Frobenius solution which would form the basis for a positive solution of (7).

Remark 1. Contrast this with the analysis of the closed Dynamic Leontief Model, i.e.

$$
x(t)=A x(t)+K[x(t+1)-x(t)]
$$

or

$$
x(t+1)=\left[I+K^{-1}(I-A)\right] x(t)
$$

${ }^{4}$ See, for example, Chapter 5 of Pasinetti [4] Chapter 2 of Woods [6]. 
Perron-Frobenius theory is applied to (10) via $\left[K^{-1}(I-A)\right]^{-1}=(I-A)^{-1} K>[0]$ from (A2). Note the implicit assumption that $K$ is non-singular, which is stronger and less realistic than (A1).

Following the terminology adopted for the Dynamic Leontief Model, (2) can be described as an open system, (7) as a closed system.

DEFINITION 1. Any sequence of semi-positive vectors, $u(t)$, satisfying (7) is called a closed solution.

Definirion 2. A closed function solution $\{u(t)\}$ is balanced if $u(t+1)=$ $\phi u(t), \phi>0$ being the growth factor.

A balanced, closed solution of (7) satisfies:

$$
B u(t)=\phi C u(t), \quad \text { or }(B-\phi C) u(t)=\underline{0} .
$$

RemarK 2. $\phi$ is thus a characteristic root of $B$ relative to $C$ or, by (A1), a characteristic root of $C^{-1} B$, with $u(t)$ the corresponding characteristic vector; clearly, $\phi^{-1}$ is a characteristic root of $B^{-1} C$.

The existence of a balanced, closed solution to (7) is the first question for consideration. (7) can be rewritten as

$$
u(t)=A u(t+1)+K[u(t+1)-u(t)]
$$

Adding $-A u(t)$ to both sides of (12), we obtain

$$
(I-A) u(t)=(A+K)[u(t+1)-u(t)] .
$$

As $(I-A)^{-1}>[0]$ by (A2), (13) can be rewritten as

$$
u(t)=(I-A)^{-1}(A+K)[u(t+1)-u(t)] .
$$

Define

$$
F=(I-A)^{-1}(A+K)>[0] .
$$

(14) can then be rewritten as

$$
(I+F) u(t)=F u(t+1) .
$$

From (A1), (A2) and (15), $F$ is non-singular; so (16) becomes:

$$
u(t+1)=\left(I+F^{-1}\right) u(t) \text {. }
$$

REMARK 3. This series of manipulations enables us to deal with the forward difference equation, (17), rather than (7). The advantage of dealing with (17), compared with (7), is that Perron-Frobenius theory can be applied. In this sense, 
the outcome of the above manipulations is analogous to the outcome of the manipulations performed on the Dynamic Leontief Model (see Remark 1).

THEOREM 1. Under (A1) and (A2)

(i) $B u=\phi C u$ has solution $\phi^{*}>1, u^{*}>\underline{0} ; p^{\prime} B=\phi p^{\prime} C$ has solution $\phi^{*}>1$, $p^{*}>\underline{0}$.

(ii) The Frobenius solution is the only semi-positive, balanced, closed solution.

(iii) $\phi^{*}<1 / \lambda^{*}(A)$.

(iv) $(B-\phi C)^{-1}>[0]$ if and only if $\phi<\phi^{*}$.

Proof. (i) By the same manipulations as above

$$
B u=\phi C u
$$

can be rewritten as

$$
\left(I+F^{-1}\right) u=\phi u .
$$

By the Perron-Frobenius Theorem

$$
\begin{aligned}
F u & =\lambda u, F>[0] \text { has solution: } \\
\lambda & =\lambda^{*}(F)>0, \quad u=u^{*}>\underline{0} .
\end{aligned}
$$

Then

$$
\left(I+F^{-1}\right) u^{*}=\left[1+1 / \lambda^{*}(F)\right] u^{*} .
$$

So

$$
\phi^{*}=1+1 / \lambda^{*}(F)>1, \quad u=u^{*}>\underline{0}
$$

solve (18). Exactly the same argument can be applied to

$$
p(t)^{\prime} B=p(t+1)^{\prime} C
$$

to yield

$$
p^{\prime} B=\phi p^{\prime} C
$$

which has solution:

$$
\phi^{*}>1, p=p^{*}>\underline{0} .
$$

(ii) Uniqueness of the balanced, closed solution follows trivially from primitivity, in fact positivity, of $F$ (see Chapter 2 of Woods [6]).

$$
F=(I-A)^{-1}(A+K)>(I-A)^{-1} A=\bar{F} .
$$

Hence

$$
\lambda^{*}(F)>\lambda^{*}(\bar{F})
$$

by a property of the Frobenius root. So

$$
1+1 / \lambda^{*}(F)<1+1 / \lambda^{*}(\bar{F})
$$


But from (26)

$$
\lambda^{*}(\bar{F})=\lambda^{*}(A) /\left[1-\lambda^{*}(A)\right] .
$$

From (22), (28) and (29)

$$
\phi^{*}<1 / \lambda^{*}(A) .
$$

(iv) Sufficiency. From (18) and (22)

$$
B u^{*}=\phi^{*} C u^{*}, \quad \phi^{*}>1 .
$$

If $\phi \in\left[1, \phi^{*}\right)$

$$
B u^{*}>\phi C u^{*} \text { or }(B-\phi C) u^{*}>\underline{0}
$$

as $u^{*}>\underline{0}$. Now

$$
\begin{aligned}
B-\phi C & =I+K-\phi(A+K) \\
& =I-\phi[A+(1-1 / \phi) K] \\
& \equiv D=\left[d_{i j}\right] .
\end{aligned}
$$

For $\phi \in\left[1, \phi^{*}\right)$

$$
d_{i j} \leqslant 0, \quad \forall i \neq j .
$$

Relations (31) and (33) imply that $(B-\phi C)^{-1}>[0]$ by a Corollary of the Hawkins-Simon Theorem (see Chapter 2 of Woods [6]).

Necessity: Suppose $(B-\phi C)^{-1}>[0]$,

$$
B-\phi C \equiv I-\phi E(\phi),
$$

where

$$
E(\phi)=A+(1-1 / \phi) K
$$

Then $(I-\phi E(\phi))^{-1}>[0]$ if and only if

$$
1 / \phi>\lambda^{*}(E(\phi))
$$

by a Corollary of the Perron-Frobenius Theorem. From (i), $(B-\phi C)$ has root $\phi^{*}$. Hence, $(I-\phi E(\phi))$ has root $\phi^{*}$. That is

$$
\lambda^{*}\left(E\left(\phi^{*}\right)\right)=1 / \phi^{*} \text {. }
$$

From (36) and (37), $\phi^{*}>\phi$, as required.

Theorem 1 establishes the existence of a balanced solution to the closed model. (iii) demonstrates the expected result that the growth rate would be greater if there were only circulating capital; for if $K=[0]$, the growth rate would be $1 / \lambda^{*}(A)-1$. This Theorem, especially (iv), will be used in the next section to derive the existence of a balanced solution to the open model. 


\section{The non-homogeneous or open system}

We now consider the open system:

$$
\begin{gathered}
B x(t)=C x(t+1)+f(t+1), \\
f(t) \geqslant \underline{0}, x(t) \geqslant \underline{0} \text { for all } t .
\end{gathered}
$$

To (2), we append

$$
B x(0)=\bar{x}(0),
$$

where $\bar{x}(0)$ is the vector of initial endowments. (38) states that the initial endowments are fully utilised.

Definition 3. A final demand sequence $\{f(t)\}$ is feasible if it satisfies (2) and (38).

Definition 4. A feasible final demand sequence is balanced if:

$$
f(t+1)=\theta f(t), \quad \theta>0 .
$$

We can immediately derive

THEOREM 2. Under (A1) and (A2), a balanced feasible final demand sequence exists if and only if $\theta<\phi^{*}$.

Proof. Sufficiency: Suppose $\theta<\phi^{*}$. Consider:

$$
x(t+1)=\theta x(t), \quad f(t+1)=\theta f(t) .
$$

Substituting into (2), we obtain

$$
(B-\theta C) x(t)=\theta f(t)
$$

whence

$$
x(t)=(B-\theta C)^{-1} \theta f(t)>\underline{0}
$$

by Theorem 1(iv). As

$$
f(t)=\theta^{t-1} f(1)
$$

(41) can be rewritten as

$$
x(t)=\theta^{\prime}(B-\theta C)^{-1} f(1)>\underline{0} .
$$

The initial endowment required for the final demand sequence is

$$
B x(0)=B(B-\theta C)^{-1} f(1) \text {. }
$$


If $\bar{x}(0)=B x(0)$, the proof is complete. If $\bar{x}(0) \neq B x(0)=B(B-\theta C)^{-1} f(1)$, define:

$$
z(0)=B^{-1} \bar{x}(0)-(B-\theta C)^{-1} f(1) .
$$

Then $\bar{x}(0)=B z(0)+B(B-\theta C)^{-1} f(1)$ and $z(\underline{0})$ is an initial point on a balanced closed solution.

Necessity: Any balanced final demand sequence with $\theta \geqslant \phi^{*}$ is not feasible by Theorem 1(iv). Necessity follows.

The analogue of Theorem 2 holds for the circulating capital model (1), as can be seen by putting $K=[0]$ and replacing $\phi^{*}$ by $1 / \lambda^{*}(A)$. Thus, the introduction of fixed capital does not qualitatively alter the results to be derived on balanced growth. It remains to be seen whether this conclusion also holds for unbalanced growth.

REMARK 4. Nikaido [3] proved for the circulating capital model (1) that convergence of either:

$$
\sum_{v=0}^{\infty} A^{v} f(v+1) \text { or } \sum_{v=0}^{\infty}\left(\lambda^{*}(A)\right)^{v} f(v+1)
$$

entailed that of the other; furthermore, that (1) has a solution if and only if ( $\alpha$ ) is convergent.

So, we now relax proportionality between final demand vectors in successive periods. The first result on unbalanced growth is

THEOREM 3. Under (A1) and (A2), a necessary condition for a feasible final demand sequence is that

$$
\sum_{v=0}^{\infty}\left(\phi^{*}\right)^{-v} f(v+1) \text { converges. }
$$

Proof. Let $\{f(t)\}$ be feasible. Then from (2)

$$
x(t+1)=C^{-1} B x(t)-C^{-1} f(t+1)
$$

or, after iteration

$$
x(t)=\left(C^{-1} B\right)^{t} x(0)-\sum_{v=1}^{t}\left(C^{-1} B\right)^{t-v} C^{-1} f(v) .
$$

Multiplying (47) by $\left(B^{-1} C\right)^{t}$, we obtain

$$
\left(B^{-1} C\right)^{t} x(t)=x(0)-B^{-1} \sum_{v=0}^{t-1}\left(C B^{-1}\right)^{v} f(v+1) \text {. }
$$


Multiplying (48) by $B$, we obtain

$$
\left(C B^{-1}\right)^{t-1} C x(t)=B x(0)-\sum_{v=0}^{t-1}\left(C B^{-1}\right)^{v} f(v+1)
$$

or, as $C=\left(C B^{-1}\right) B$ :

$$
\left(C B^{-1}\right)^{t} B x(t)=B x(0)-\sum_{v=0}^{t-1}\left(C B^{-1}\right)^{v} f(v+1) .
$$

From Theorem 1(i)

$$
p^{* \prime} B=\phi^{*} p^{* \prime} C, p^{*}>\underline{0} \text { or }\left(\phi^{*}\right)^{-1} p^{* \prime}=p^{* \prime} C B^{-1} \text {. }
$$

Premultiplying (49) by $p^{*}$ and using (50), we obtain

$$
\left(\phi^{*}\right)^{-1} p^{* \prime} B x(t)=p^{* \prime} B x(0)-p^{* \prime} \sum_{v=0}^{t-1}\left(\phi^{*}\right)^{-v} f(v+1) .
$$

As the left-hand side of (51) is non-negative, it follows that:

$$
p^{* \prime} B x(0) \geqslant p^{* \prime} \sum_{v=0}^{t-1}\left(\phi^{*}\right)^{-v} f(v+1) .
$$

As $p^{*}>\underline{0}$, convergence of the sum follows. This completes the proof.

We require Theorem 3 in the proof of

THEOREM 4. Under (A1) and (A2), if $x(t)$ is a feasible solution of (2) and (38) and $u(t)$ is a closed solution:

$$
\lim _{t \rightarrow \infty}\left(\phi^{*}\right)^{-t}[x(t)-u(t)]=\underline{0} .
$$

Proof. From (2)

$$
\left(\phi^{*}\right)^{-t}[B x(t)-C x(t+1)]=\left(\phi^{*}\right)^{-t} f(t+1) .
$$

By Theorem 3

$$
\lim _{t \rightarrow \infty}\left(\phi^{*}\right)^{-t} f(t+1)=\underline{0} .
$$

So, we have

$$
\lim _{t \rightarrow \infty}[B x(t)-C x(t+1)]=\underline{0} .
$$

There are two cases to consider

(i) $\lim _{t \rightarrow \infty}\left(\phi^{*}\right)^{-t} x(t)=\underline{0}$. As $u(t)=\underline{0}$ solves (7), (52) clearly holds for this choice of $u(t)$. 
(ii) $\lim _{t \rightarrow \infty}\left(\phi^{*}\right)^{-t} x(t) \geqslant \underline{0}$. As $u(t)$ solves (7), we have after iteration

$$
u(t)=\left(C^{-1} B\right)^{t} u(0) \text {. }
$$

Premultiplying by $\bar{p}>\underline{0}$ ( $\bar{p}$ to be determined below):

$$
\bar{p}^{\prime} u(t)=\bar{p}^{\prime}\left(C^{-1} B\right)^{t} u(0) \text {. }
$$

From Theorem 1, $p^{*}$ satisfies:

$$
p^{* \prime} B=\phi^{*} p^{* \prime} C \quad \text { or } \quad p^{* \prime} C C^{-1} B=\phi^{*} p^{* \prime} C \text { or } \quad \bar{p}^{\prime} C^{-1} B=\phi^{*} \bar{p}^{\prime}
$$

where $\bar{p}^{\prime}=p^{* \prime} C>\underline{0}^{\prime}$. Using (59) in (58), we have:

$$
\bar{p}^{\prime} u(t)=\left(\phi^{*}\right)^{t} \bar{p}^{\prime} u(0) \text {. }
$$

As $u(0)$ is finite, it follows from $(60)$ that $\left(\phi^{*}\right)^{-t} u(t)$ is bounded. Given $\bar{x}(0) \geqslant \underline{0}$, it is possible to find $u(t)$ such that:

$$
u(t) \geqslant x(t)
$$

by using (2) and (7). Hence, $\left(\phi^{*}\right)^{-t} x(t)$ is bounded. For an appropriate subsequence $\left\{t_{\nu}\right\}$ of $\{t\}$

$$
\lim _{t_{v} \rightarrow \infty}\left(\phi^{*}\right)^{-t_{v}}\left[B x\left(t_{v}\right)-C x\left(t_{v}+1\right)\right]=\underline{0}
$$

or

$$
B \lim _{t_{\nu} \rightarrow \infty}\left(\phi^{*}\right)^{-t_{\nu}} x\left(t_{\nu}\right)=\phi^{*} C \lim _{t_{\nu} \rightarrow \infty}\left(\phi^{*}\right)^{-\left(t_{\nu}+1\right)} x\left(t_{\nu}+1\right) .
$$

The limits are used to define a closed solution $u(t)$ such that

$$
\lim _{t \rightarrow \infty}\left(\phi^{*}\right)^{-t}[x(t)-u(t)]=\underline{0} .
$$

This completes the proof.

It is a straightforward matter to establish that, if $y(t)$ is a particular solution of (2) and $u(t)$ is a closed solution, $x(t)=u(t)+y(t)$ is the general solution of (2). Theorem 3 tells us that the particular solution, $y(t)$, is minorant to any solution, $x(t)$. This is easy to see in the case of a balanced final demand sequence, as shown by Theorem 2. i.e.

$$
\lim _{t \rightarrow \infty}\left(\phi^{*}\right)^{-t} y(t)=\lim _{t \rightarrow \infty}\left(\phi^{*}\right)^{-t} \theta^{t}(B-\theta C)^{-1} f(1)=\underline{0}
$$

as $\theta<\phi^{*}$. Theorem 4 extends this result to cases where the final demand vector does not grow in a balanced way and the appropriate solutions exist.

REMARK 5. Further to Remark 4, Nikaido [3] proves that the circulating capital model (1) has solution

$$
x(t)=u(t)+\sum_{v=0}^{\infty} A^{v} f(v+t+1)
$$


$u(t)$ being a solution of the homogeneous equation

$$
x(t)=A x(t+1) \text {. }
$$

He proves also that

$$
\lim _{t \rightarrow \infty}\left(\lambda^{*}(A)\right)^{t} y(t)=\underline{0}
$$

where $y(t)=\sum_{v=0}^{\infty} A^{v} f(v+t+1)$.

It is evident that Theorem 4 provides an extension of Nikaido's result to our fixed capital model.

We require Theorems 3 and 4 in the proof of the main result on non-proportional growth:

Theorem 5. Under (A1), (A2) and $\left|\lambda_{l}\left(B^{-1} C\right)\right| \leqslant\left(\phi^{*}\right)^{-1}$ for all $i$, a necessary condition for a feasible final demand sequence is that

$$
\sum_{v=0}^{\infty}\left(C B^{-1}\right)^{v} f(v+1) \text { converges. }
$$

Proof. From Theorem 4

$$
\lim _{t \rightarrow \infty}\left(\phi^{*}\right)^{-t}[x(t)-u(t)]=\underline{0} .
$$

From (2)

$$
x(t)=B^{-1} C x(t+1)+B^{-1} f(t+1)
$$

or, after premultiplying by $\left(B^{-1} C\right)^{t}$

$$
\left(B^{-1} C\right)^{t} x(t)=\left(B^{-1} C\right)^{t+1} x(t+1)+\left(B^{-1} C\right)^{t} B^{-1} f(t+1) .
$$

From (7)

$$
u(0)=\left(B^{-1} C\right)^{t+1} u(t+1) .
$$

Subtracting (68) from (69), we obtain after rearranging

$$
\begin{aligned}
\left(B^{-1} C\right)^{t} x(t)= & u(0)+\left(B^{-1} C\right)^{t+1}[x(t+1)-u(t+1)] \\
& +\left(B^{-1} C\right)^{t} B^{-1} f(t+1) \\
= & u(0)+\left(\phi^{*} B^{-1} C\right)^{t+1}\left(\phi^{*}\right)^{-(t+1)}[x(t+1)-u(t+1)] \\
& +\left(\phi^{*} B^{-1} C\right)^{t} B^{-1}\left(\phi^{*}\right)^{-t} f(t+1) .
\end{aligned}
$$

By hypothesis, the characteristic roots of $B^{-1} C$ satisfy

$$
\left|\lambda_{\imath}\left(B^{-1} C\right)\right| \leqslant\left(\phi^{*}\right)^{-1} \text {. }
$$


It follows that

$$
\lim _{t \rightarrow \infty}\left(\phi^{*} B^{-1} C\right)^{t}=M, \text { a constant matrix. }
$$

From Theorem 4

$$
\lim _{t \rightarrow \infty}\left(\phi^{*}\right)^{-(t+1)}[x(t+1)-u(t+1)]=\underline{0} .
$$

From Theorem 3

$$
\lim _{t \rightarrow \infty}\left(\phi^{*}\right)^{-t} f(t+1)=\underline{0} .
$$

Using (71)-(73) in (70), we have

$$
\lim _{t \rightarrow \infty}\left(B^{-1} C\right)^{t} x(t)=u(0) \geqslant \underline{0} .
$$

Applying (74) to (48), we obtain

$$
\begin{aligned}
\lim _{t \rightarrow \infty}\left(B^{-1} C\right)^{t} x(t) & =u(0) \\
& =x(0)-B^{-1} \sum_{v=0}^{\infty}\left(C B^{-1}\right)^{v} f(v+1) .
\end{aligned}
$$

This completes the proof.

There are obvious differences between Nikaido's results (mentioned in Remarks 4 and 5) on the circulating capital model (1), and our results on the fixed capital model (2). First, Nikaido derives necessary and sufficient conditions for a solution to (1), whereas we are able to derive only necessary conditions for a solution to (2). This difference can be attributed to the presence of the matrix $B$. As this matrix is not in general diagonal, its inverse contains negative entries; $C B^{-1}$ may also contain negative entries. Nikaido's proof relies on the presence of semi-positive vector series, such as $(\alpha)$ and $(\beta)$ in Remark 4; this point is demonstrated by the following

Corollary 1. If $B^{-1} \sum_{v=0}^{\infty}\left(C B^{-1}\right)^{v-t} f(v+1) \geqslant \underline{0}$ for all $t$, (67) is also sufficient for a feasible final demand sequence.

Proof. From the proof of Theorem 5, we have

$$
\begin{aligned}
u(t) & =\left(C^{-1} B\right)^{t} u(0) \\
& =x(t)-B^{-1} \sum_{v=0}^{\infty}\left(C B^{-1}\right)^{v-t} f(v+1)
\end{aligned}
$$


because the choice of the initial period is arbitrary. As $u(t) \geqslant \underline{0}$ by (74), we have from (76)

$$
x(t)=u(t)+B^{-1} \sum_{v=0}^{\infty}\left(C B^{-1}\right)^{v-t} f(v+1) .
$$

The result now follows.

There is a second difference worth noting-a characteristic root assumption is present in Theorem 5 but is absent from Nikaido's results. To appreciate the role of this assumption, suppose that there exists a root of $B^{-1} C$, denoted by $\lambda_{j}$, such that $\left|\lambda_{J}\right|>\left(\phi^{*}\right)^{-1}$. Consider the balanced final demand sequence given by (39). From Theorem 2, this sequence is feasible if $\theta<\phi^{*}$, when:

$$
\begin{aligned}
x(t) & =\theta^{t}(B-\theta C)^{-1} f(1) \\
& =\theta^{t} B^{-1}\left(I-\theta C B^{-1}\right)^{-1} f(1) .
\end{aligned}
$$

$\left(I-\theta C B^{-1}\right)^{-1}=\sum_{v-0}^{\infty}\left(\theta C B^{-1}\right)^{v}$ if and only if $1>\left|\lambda_{l}\left(\theta C B^{-1}\right)\right|=\theta\left|\lambda_{l}\left(C B^{-1}\right)\right|$. i.e. $\theta^{-1}>\left|\lambda_{l}\left(C B^{-1}\right)\right|$. Hence, if there exists $\lambda_{\text {, such that }}\left|\lambda_{j}\right|>\theta^{-1}>\left(\theta^{*}\right)^{-1}$, the series $\sum_{v=0}^{\infty}\left(C B^{-1}\right)^{v} f(v+1)$ does not converge for an appropriate choice of $f(1)$; that is, if $f(1)$ is expressed as a linear combination of characteristic vectors including that corresponding to $\lambda$, There is an obvious extension if there is more than one root such that $\left|\lambda_{j}\right|>\left(\phi^{*}\right)^{-1}$. So, we have this negative result.

COROLlaRY 2. If the characteristic root condition of Theorem 5 does not hold, convergence of $\sum_{v=0}^{\infty}\left(C B^{-1}\right)^{v} f(v+1)$ is not necessary for a feasible final demand sequence.

\section{Conclusions}

We have remarked above on the similarity between the circulating and fixed capital models when the final demand sequence is balanced. For the former, the inverse of the Frobenius root of $A$ and for the latter, $\phi^{*}$ (the inverse of the quasi-Frobenius root of $B^{-1} C$ ) provide the upper limit to the growth factor. This similarity is a consequence of the type of analysis performed-that is, balanced growth analysis-on the type of model under examination-both the circulating and fixed capital models are essentially of the single-product industries variety. In balanced growth analysis, it suffices to consider the model in two consecutive periods; the growth factor can then be inferred. Once the growth path has been chosen, there is no reason to alter the proportions between the quantities produced. 
It is when the final demand sequence is unbalanced that there is a divergence between the properties of the circulating and fixed capital models. From the mathematical point of view, this divergence can be attributed to the presence of the semi-positive, non-diagonal matrix $B$, which prevents the direct application of Nikaido's analysis of the circulating capital model to the fixed capital case. From the economic point of view, there is a corresponding inherent difference. In a circulating capital model, there are only flow conditions to be satisfied; in a fixed capital model, there are in addition stock conditions to be satisfied. Therefore, it is to be expected that more stringent (necessary and sufficient) conditions will be required for the fixed capital model than for the circulating capital model or that conditions which are necessary and sufficient for the latter will have analogues which are only necessary for the latter.

\section{Acknowledgements}

Non-incriminatory acknowledgements are due to the referees and the editor for criticism of the first version of this paper.

\section{References}

[1] M. Morishima, The theory of economic growth (Oxford, Clarendon Press, 1969).

[2] J. von Neumann, "A model of general economic equilibrium", Rev. Econom. Stud. 13 (1945-6), 1-9.

[3] H. Nikaido, Convex structures and economic theory, (London, Academic Press, 1968).

[4] L. L. Pasinetti, Lectures on the theory of production (London, Macmillan, 1977).

[5] P. Sraffa, Production of commodities by means of commodities (Cambridge, Cambridge University Press, 1960).

[6] J. E. Woods, Mathematical economics (London, Longman Group, 1978).

[7] J. E. Woods, "Notes on Sraffa's fixed capital model", J. Austral. Math. Soc. Ser. B 26 (1984), 200-232. 